\title{
Racial Disparities in Depression Care among Older Adults: Can the Perspectives of Clinicians and Patients be Reconciled?
}

\author{
Karen A. Zurlo • Colleen M. Beach
}

Published online: 8 January 2013

(C) Springer Science+Business Media New York 2013

\begin{abstract}
Depression is highly prevalent and debilitating in late life. It affected 3.8 million older Americans in 2005, and its prevalence is expected to increase to 6.6 million in 2025. Despite its prevalence and associated negative health outcomes, depression is not diagnosed and treated equally among older Americans. An under-recognition and undertreatment of depression in minority elders exists, which has lead to significant disparities between Whites and nonWhites. These disparities challenge our principles of equality, equity and adequacy and, in their most extreme form, become human rights issues. As a result, eliminating health disparities has become a priority of the US Federal government. Practitioners must address differences in the diagnosis and treatment of depression among clients served. Increased levels of cultural competency and educating clients will help reconcile differences between clinicians and their patients and lead to increased understanding of client needs, while decreasing disparities in depression care and diagnosis.
\end{abstract}

Keywords Depression - Disparities · Differences · Older adults · Elderly $\cdot$ Treatment $\cdot$ Diagnosis $\cdot$ Clinicians $\cdot$ Patients Mental health care - Aging - Minorities $\cdot$ Race $\cdot$ Discrimination . Communication $\cdot$ Education $\cdot$ Stigma $\cdot$ Culture

\footnotetext{
K. A. Zurlo $(\square)$

School of Social Work, Rutgers, The State

University of New Jersey, 536 George Street,

New Brunswick, NJ 08901, USA

e-mail: kzurlo@ssw.rutgers.edu

C. M. Beach

Institute for Families, School of Social Work, Rutgers,

The State University of New Jersey, 390 George St.,

6th Floor, New Brunswick, NJ 08901, USA

e-mail: cbeach@ssw.rutgers.edu
}

\section{Introduction}

Depression is highly prevalent and debilitating in late life and predicts a range of negative health outcomes. In 2005, it affected 3.8 million older Americans [1••]. As the baby boom population ages, it is anticipated that the impact of depression on this growing older adult population will increase proportionally. The number of people older than 65 years with psychiatric disorders in the US is projected to increase to about 8.2 million in $2050[1 \bullet \cdot$, where the increase in depression symptomatology will be most pronounced among older men $[1 \bullet \bullet]$. Although progress has been made in characterizing the presentation of late-life depression and in improving treatment, it continues to have detrimental consequences that include family disruption, the worsening of medical illness and disability, and increasing mortality $[2,3]$. Moreover, major depression is a leading cause of disability and accounts for more than half the suicides that occur annually [4]. The current health care system serving older adults with mental illness is inadequate and unprepared to meet the upcoming crisis in geriatric mental health $[5 \bullet \cdot]$.

Moreover, depression is not diagnosed and treated equally among older Americans. Studies indicate that there is continued under-recognition and under-treatment of depression in minority elders $[5 \bullet \bullet, 6 \bullet, 7,8]$, leading to significant disparities in diagnosis and treatment between Whites and non-Whites. These disparities challenge our principles of equality, equity and adequacy that are at the core of our national identity. As a priority of the US Federal government, efforts to reduce and eliminate mental health disparities are gaining momentum [9].

It is only in the past decade that addressing differences in mental health care has become a national priority. While the term, disparities, is not standardized, it generally implies an inequity or an injustice rather than a simple inequality [10]. The World Health Organization defines disparities as 
"differences in health that are unnecessary, avoidable, unjust, and unfair" [11]. In a seminal work, the Institute of Medicine defined disparities as "racial or ethnic differences in the quality of healthcare that are not due to access-related factors or clinical needs, preferences, and appropriateness of intervention" [12••]. The Joint Center for Political and Economic Studies reported that "the combined costs of health inequalities and premature death in the United States were $\$ 1.24$ trillion between 2003 and 2006" [13]. Hence, the costs to a society are exorbitant. Not only are lives lost unnecessarily, but disparities often lead to charges of discrimination and, in the extreme, can be considered a human rights issue. To eliminate disparities, research is needed that provides insight into such questions as: How and why do racial and ethnic disparities occur? How do the patient and practitioner contribute to mental healthcare disparities? Do bias, stereotyping, prejudice and poor communication exacerbate these disparities? The studies reviewed in this paper address these and other related questions.

\section{Background on Depression}

Depressive symptoms are an important indicator of general well-being and mental health among older adults [14]. Adults who report depressive symptoms often experience higher rates of physical illness, greater functional disability and higher health care resource utilization [15]. Moreover, depressive conditions cause substantial disability, and the costs incurred are substantial as well. In the US alone, in 1990, the cost of depressive conditions was $\$ 43.7$ billion/year, where, $\$ 12.4$ billion $(28 \%)$ was attributable to direct costs, $\$ 7.5$ billion (17\%) comprises mortality costs, and $\$ 23.8$ billion (55\%) was derived from the two morbidity cost categories [16]. These costs have risen dramatically in the recent decade due to the increased rates of diagnosis and the corresponding increased use of anti-depressants. Also, the Surgeon General reported that racial and ethnic minorities bear a greater burden from unmet mental health needs and thus suffer a greater loss to their overall health and productivity [17].

In addition to the emotional strain experienced by those diagnosed with depression, depression predicts a range of negative health outcomes, including total mortality, suicide, and hospitalization, while reducing active life expectancy (at age 70 years) by 6.5 years for men and 4.2 years for women [14]. If untreated or undertreated, depression can significantly diminish quality of life and increase morbidity and mortality among the elderly. Depression also complicates several comorbid general medical conditions that are common in older populations, such as congestive heart failure, diabetes, and arthritis [17]. Major depression is a prevalent disorder and a major public health concern in the US. In 2008, $11 \%$ of men and $16 \%$ of women aged 65 years and older experienced clinically relevant depressive symptoms [14].
Effective treatment exists. Antidepressant treatment and psychotherapy, particularly in combination, have been shown to be effective in increasing rates of remission. However, trends in the diagnosis and treatment of depression suggest that depression is under-diagnosed and undertreated in older adult members of ethnic and racial minority groups $[1 \bullet, 2]$.Understanding these trends is an essential prerequisite to targeting solutions to the affected groups.

\section{Depression and the Elderly}

To understand racial and ethnic disparities in depression treatment and diagnosis among the elderly, it is also essential to take into account other demographic characteristics of the population including age differentials, marital status, education, religious preferences, health status, veteran status, and income levels, to name a few. It is in the best interest of policy makers, researchers, administrators and practitioners to understand the characteristics of the elderly population in as much detail as possible, in order to make an accurate assessment and propose appropriately individualized diagnoses and treatments.

In 2008, the proportion of people age 65 years and over with clinically relevant symptoms was higher for people age 85 years and over $(18 \%)$ than for people in any of the younger groups (12-15\%) [14]. Also, Sonnenberg, and colleagues [18] conducted a longitudinal study that investigated antidepressant use from 1992 to 2002 in a population-based sample aged $65-85$ years. The authors found that antidepressant use increased from $2 \%$ to $6 \%$. And in the group with major depressive disorder, treatment with antidepressants showed an increase from $15 \%$ to $30 \%$, where the increase was larger in the older old than in the younger old.

In an older study, Blazer and colleagues [19] studied race as a correlate of medication use in a sample of older adults. They found that, in 1986, a total of $4.6 \%$ of the Whites and $2.3 \%$ of the African Americans in the sample used antidepressants. By 1996, utilization of antidepressants by the Whites in the sample had increased to $14.3 \%$ while, for African Americans, it had increased to $5.0 \%$. Though antidepressant use had increased for both groups during the 10-year interval, the increase was far smaller for the African American elders.

Summarized below are several more recent studies which, taken together, support the conclusion that mental health disparities are more severe among older adults than in the general population, and are compounded by being at higher risk for misdiagnosis, under-treatment, and more severe depressive symptomatology than Whites. [20]. In treatment, older members of ethnic and racial minority groups are more likely to be given first-generation drugs and fewer prescriptions for similar diagnoses than their White counterparts [20]. 


\section{Disparities in Depression Care}

To understand disparities in the treatment of depression, it is important first to note racial and ethnic differences in rates of diagnosis of depression among older Americans. Akinci-

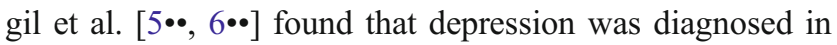
the elderly with increasing frequency between 1992 and 2006; between the years 1992 - 1995 and 2002 - 2005, the rate rose from $3.2 \%$ to $6.3 \%$. Trends in antidepressant use increased in these same periods from $7.3 \%$ to $15.3 \%$ among older adults $[5 \bullet \bullet, 6 \bullet \cdot$. Yet the trend was not consistent among races and ethnicities. Compared with Blacks and Hispanics, Whites were found to have higher rates of diagnosis and treatment with antidepressants.

A secondary analysis of data from the 2005 California Health Interview Survey used a population-based sample of older adults to examine racial and ethnic differences in the prevalence rates of psychological distress and the reported need and use of mental health services [17]. The sample population comprised 16,974 people aged 55 years and older, with 13,974 non-Latino Whites, 719 African Americans, 1,215 Asians, and 1,066 Latinos. The authors found that African Americans, Asians, and Latinos were more likely than Whites to report mental distress (21.2-24.2\% vs. $14.4 \%, P<0.001$ ) [21]. Moreover, all three groups had worse access to mental health services than Whites (African American $\mathrm{aOR}=0.64,95 \% \mathrm{CI}=0.43-0.96$; Asian $\mathrm{aOR}=$ $0.32, \mathrm{CI}=0.16-0.63$; Latino $\mathrm{aOR}=0.35, \mathrm{CI}=0.17-0.70)$.

Using a nationally representative sample $(N=8762)$, Alegria et al. [7] found similar differences between ethnic/ racial minority patients and non-Latino Whites with respect to access to and quality of depression treatments. Their results indicated that for those with last year depressive disorder, $63.7 \%$ of Latinos, $68.7 \%$ of Asians, and $58.8 \%$ of African Americans, vs. $40.2 \%$ of non-Latino Whites, did not access any last year mental health treatment $(P<0.001)$.

In a sample of over 85,000 primary care outpatients residing in the Northeast (Connecticut, New York, Rhode Island, New Hampshire, Massachusetts), Trinh et al. [18, 22] found that minority groups were less likely to be diagnosed with depression than Whites. Of the Whites, $11.36 \%$ were diagnosed with depression; for Asian Americans, African Americans, and Latino Americans, depression diagnosis rates were $6.44 \%, 7.55 \%$ and $10.18 \%$, respectively. Protective factors that may help explain these differences include religiosity and spiritual beliefs, as well as limited cultural understandings between the practitioner and client.

When comparing Whites and non-Whites on depression severity, Gonzalez, Tarraf, Whitfield, and Vega [23] found that major depression chronicity was higher among Mexican Americans, Puerto Ricans and African Americans than among Whites. Secondly, among those meeting 12-month major depression criteria, Vietnamese, Mexican and African
Americans were the least likely to receive APA Guideline concordant depression therapy.

Traditional treatment of depression consists of three main modalities: antidepressant use, psychotherapy and the two in combination. A number of studies illustrate that there are disparities in treatment. Whites are more likely to be treated with antidepressants than are Blacks. Another form of treatment that has not received much recognition in the literature is the use of electroconvulsive therapy (ECT). Blacks with depression were less likely to receive ECT than Whites during the 1970s and 1980s. Case et al. [24] examined whether this racial difference in receiving ECT persists. Their results indicated that the disparities persist. Depressed Black inpatients are less likely than Whites to receive ECT; the difference arises almost entirely from the lesser use of ECT in hospitals where it is available.

Yet one complication that impedes identification of disparities in treatment access and quality is the fact that much survey data provide insufficient respondent data by ethnic subgroups [23]. In many large national surveys, the nonWhite population totals $10 \%$ of the sample, leaving insufficient effect size to draw conclusions from the survey data. Additionally, many surveys do not segregate the race and ethnicity of the respondents in more distinct ways, other than identifying as Black, Hispanic, or Asian. This, too, limits the ability to draw any viable conclusions on nonWhite populations.

Even so, the studies reviewed support the conclusion that, compared with Whites, African American seniors are underdiagnosed and under-treated for depression. Some plausible explanations exist. To address treatment disparities, the practitioner must understand concerns and preferences of individuals from diverse cultural groups $[25,26]$. Perceptions and definitions of depression may vary between African Americans and Whites. By examining beliefs regarding mental health, the practitioner will be better equipped to understand and diagnose a mental health condition.

\section{Cultural Issues}

Understanding the concerns and preferences of individuals in diverse cultural groups is critical to their care because unfamiliarity with the patient's culture can act as a barrier to mental health treatment $[27,29]$. In physical healthcare as well as in mental healthcare, patients bring to the healthcare encounter cultural backgrounds, beliefs, practices, and languages that require culturally competent communication to maximize the quality of care they receive [30]. Attunement to cultural differences may be the first step in providing culturally competent care.

Everyone has a culture of origin, which directly affects how one interacts with members of one's own group and 
with members of other groups. Cultural background can affect values, beliefs and communication styles. Particularly in the health care setting, the consequences of interaction (e.g., between clinician and patient) can be very serious. Inability to recognize or accept differences between oneself and one's patient may affect how well instructions are communicated to the patient, or subconsciously affect clinical decision making [30]. The extent to which a patient's needs are met will depend, at least in part, on the clinician's awareness of and flexible accommodation to cultural difference $[12 \cdot \bullet]$. Investigators have found subtle effects of racial difference on clinician-patient interaction [12••]. Where the race of the physician and patient was the same, visits were longer; in addition, patients in race-concordant visits were more satisfied than patients in race-discordant visits [27, 28]. Hence, culturally competent practice plays a critical role in the understanding and treatment of depression in ethnic minorities. As a result of these findings, in the upcoming publication of the Diagnostic and Statistical Manual of Mental Disorders V (DSM-V) in 2012 substantial revisions are planned to the content of Cultural Formulation; practitioners are asked to operationalize a more thorough evaluation of the socio-cultural context in which the illness (i.e. mental health) experience is embedded [29]. A systematic cultural assessment will be recommended during the encounter between practitioner and client, where the goal is a more inclusive nosology that reminds clinicians and researchers to take culture and context into account during the diagnostic and treatment phases. As a result, conducting a cultural assessment will become good clinical practice. In sum, patient beliefs about mental health must be accurately identified and addressed in the care process [30].

\section{Workforce Issues}

In 2008, as the nation's 78 million baby boomers began to retire, the Institute of Medicine (IOM) issued a report [12 • ] concluding that the healthcare workforce is not prepared to offer care to the growing number of older patients. The number of physicians, nurses, social workers, occupational therapists and physical therapists in training and practice was projected to be insufficient to meet a growing need of practitioners. Existing disparities in mental health care for older adults - exacerbated by tenuous relationships between minority populations and medical professionals, and by reluctance to seek mental health treatment in particular may be widened still further by the impending shortage of clinicians. That the need to address these disparities is urgent is evident from reports that shortages are expected as soon as 2020. The Center for Workforce Studies (2012) [32・•] highlighted several areas of concern regarding the growing gap between physician supply and demand for services, and made recommendations to improve efficiency within the healthcare system, reconfigure healthcare delivery, and make better use of physicians and other healthcare professionals. The Center further recommended that the Federal Government take an integrated approach to plan for changes in health workforce is [32••].

Changes in the recruitment, admittance and training of individuals in the profession of medicine can be made so society is better prepared to meet the rising needs of mental health care, thus offering some protection for the underserved. For example, medical education accrediting bodies have mandated that the educational experience include training for the care of patients from varied cultural, socioeconomic, and ethnic backgrounds [33]. Cultural competency is included in the curriculum. Better attunement of the medical community to the needs of currently underserved populations could ameliorate the mistrust and miscommunication between patients and physicians.

In response to recognition of depression as a growing public health problem, the Task Force on Community Preventative Services suggests that both home-based and clinic-based depression care management are appropriate and should be encouraged for individuals over 60 years (CDC Promotes Public Health Approach to Address Depression Among Older Adults) [34]. Implementing such changes at the community and public health level might not only improve care and access to care for depressed older African Americans, but also reduce symptom severity and the demand for physicians. More creative use could be made of strengths and resources already present in underserved populations, such as their spirituality and affiliation with African American clergy and church communities, which will be discussed further below. Community-based mental health settings also could do more to develop peer support roles and skills (e.g. by inviting co-facilitation of support groups). Many more individuals might be reached through partnerships of community health, academic institutions and religious institutions.

\section{A Focus on Spirituality}

Religion and spirituality can affect health positively. In a number of recent studies, researchers found that everyday religious experiences help people cope better with stress $[35,36]$. In a study of 92,500 postmenopausal women, those who reported attending religious services were $27 \%$ less likely to have depressive symptoms than women who did not attend services [37••]. Additionally, spirituality, regardless of one's faith, is also known to boost mental health. Johnstone and colleagues [38] found that being spiritual decreased a person's sense of self in a positive manner, which enabled them to feel more connected to the world. 
Although it is unclear how religious beliefs directly affect mental health, these studies affirm that there is a positive association between the two constructs. As a result, it is recommended that practitioners be aware of the benefits of having a spirituality and become more comfortable broaching the topic of spirituality with the clients they treat.

For example, some African American patients may not believe there is a biological basis to depression and, consequently, may prefer that treatment consist of counseling and prayer. In research by Wittink, Joo, Lewis, and Barg [39] older African Americans claimed that depression was due to a "loss in faith" and faith and spiritual/religious activities could empower an individual by providing strength for healing to occur. Cultural differences in the way depression symptoms are manifested, defined, interpreted and labeled may explain, at least in part, differences in help-seeking behaviors. Blacks are less likely to seek mental health services than their White counterparts [30] and, when they seek services, they attend fewer sessions and are more likely to terminate treatment prematurely [40]. Blacks also are more likely to use informal networks, such as their church, when seeking support, without presenting themselves as depressed. Instead, they may seek friendship and general social connections. These examples illustrate why it is central to patient-centered care that patient beliefs about mental health be accurately identified and addressed in the care process [29]. Shellman and colleagues [41] concurred that there are barriers to the identification and treatment of depression in Black older adults that include the lack of access to quality mental health care, the stigma of mental illness [31], mistrust of mental health providers, and poor provider-client communication.

An approach that might be particularly effective in African American communities might be for providers of medical and mental health care to partner with churches. Gullum [42], for example, has recommended that the church community be the site of intervention and prevention efforts regarding intimate partner violence in the African American community. Though it may not be possible to stretch church resources to add to ongoing programs, low-cost interventions such as timely referral and other aspects of partnering could prove to be just as critical [32・•]. Listed below are steps (adapted from Gullum's recommendations religious institutions) which, if taken, might help to meet some of the needs of African Americans affected by depression.

- Provide education and training to clergy and lay church leaders on depression, to raise awareness and facilitate a meaningful, appropriate response;

- Educate clergy and lay leaders about community resources, such as [fill in a couple of examples];

- Partner with religious institutions to offer support groups facilitated by mental health providers or students in field placements (with appropriate supervision) and supplemented with child care services;

- Offer support groups not only for older adults with depression but also for the adult children who care for them; and

- Provide churches with informative articles for their newsletters or bulletins, to raise awareness of depression and describe the partnership of the church with other community institutions to meet the needs of the faithful.

Joining forces in this fashion, to eliminate barriers such as lack of material resources, failed communication, limited understanding of illness management, and social stigma, could be a start to alleviating racial disparities in mental health care.

\section{Discussion}

The literature presented supports the findings that disparities in depression diagnosis and treatment exist. As a consequence of these disparities, health care policy makers, researchers and practitioners must seek knowledge and clinical methods that lessen and ultimately eliminate these disparities. This paper underscores the importance that it is possible to reconcile perspectives between the clinicians and their clients. To reconcile these perspectives, we recommend institutional changes as well as the use of a more client-centered approach during the practitioner's encounter with the client. The institutional changes pertain to workforce issues and the training and development of more minority practitioners. This will foster more concordant client encounters and minimize the cultural dissonance between client and clinician, leading to more equality and equity in the treatment and diagnosis of depression among elder adults. We also recommend that clinician's pursue a more open and thorough understanding of the client's cultural context in the clinical encounter. The importance of the Cultural Formulation will be documented in the DSM V and should act as a guide for all clinicians in the diagnosis and treatment of depression in their clients. For example, depression can be considered a psychological construct that is defined differently by people of different ethnicities. The spiritual beliefs of the client may play a role in how the client presents depressive symptoms as well as how that client may prefer to be treated. Although spirituality and religiosity are relatively new phenomena in the mental health literature, there are findings that support the association between spirituality and mental health. Consequently, it is incumbent upon the practitioner to understand the client's spiritual life and where possible, use it as a resource in the treatment of depression. Because spiritual beliefs can be a psychological strength to individuals with depression, they should not be ignored in the clinical encounter. Yet, 
clinicians must be trained to assess a client's spiritual practice and learn how to discern whether an individual's spirituality can be used as a positive force in dealing with depressive symptomatology.

\section{Conclusion}

The research reviewed here attests to the depth and breadth of the disparities between Whites and racial and ethnic minorities in the diagnosis and treatment of depression. To effectively reduce and ultimately eliminate these disparities, it is recommended that the education and training of mental health professionals be revised to better prepare them for practice in diverse communities. It may be particularly important not only to educate clients about depression but also to invite clinicians and clinicians-in-training to discuss their ambivalence about religion and spirituality so that, when appropriate in treatment, they can reframe spirituality as a strength and a resource. Programs that train mental health professionals also should increase their efforts to recruit minority students. Taking these steps will increase the likelihood that older adults with depression will receive better diagnosis and treatment, thereby reducing or even eliminating disparities in mental health care.

Disclosure No potential conflicts of interest relevant to this article were reported.

\section{References}

Papers of particular interest, published recently, have been highlighted as:

-. Of major importance

1. • Heo M, Murphy CF, Fontaine KR, Bruce ML, Alexopoulos GS. Population projection of US adults with lifetime experience of depressive disorder by age and sex from year 2005 to 2050 . Int J Geriatr Psychiatry. 2008;23(12):1266-70. These authors used a nationally representative survey to estimate the prevalence of doctor-diagnosed depressive disorders from 2005 to 2050. They claimed that by the year 2050 approximately 46 million adults aged 18 or older will be diagnosed with depressive disorder. The increase in number will be more pronounced in adults aged 65 or older. The authors concluded that prevention, detection, and treatment of depressive disorders might attenuate the magnitude of this growing estimate.

2. Alexopoulos GS. Depression in the elderly. Lancet. 2005;365:1961-70.

3. Blazer DG. Depression in late life: Review and commentary. J Gerontol Med Sci. 2003;56A:249-65.

4. US Department of Health and Human Services. The road ahead: Research partnerships to transform services. A report by the national advisory mental health Council's workgroup on services and clinical epidemiology research, national institutes of health. Bethesda, MD: National Institute of Mental Health; 2006.

5. - Akincigil A, Olfson M, Siegel MJ, Zurlo KA, Walkup JT, Crystal S. Racial and ethnic disparities in depression care in community-dwelling elderly in the United States. Am J Public Health. 2012;102(2):319-28. These authors used the Medicare Current Beneficiary Survey (MCBS) and investigated racial and ethnic disparities in the diagnosis and treatment of depression among community dwelling-elderly. A critical finding in this study showed that depression diagnosis rates were $6.4 \%$ for nonHispanic whites, $4.2 \%$ for African Americans, and $7.2 \%$ for Hispanics. Their results indicate that significant raciallethnic differences exist in the diagnosis and treatment of depression in community-swelling elderly.

6. •- Akincigil A, Olfson M, Walkup JT, Siegel MJ, Kalay E, Amin S, Zurlo KA, Crystal S. Diagnosis and treatment of depression in older community-dwelling adults: 1992-2005. Journal of the American Geriatric Society. 2011;59:1042-51. doi:10.1111/j.15325415.2011.03447.x. These authors used the Medicare Current Beneficiary Survey (MCBS) and examined evolving patterns of depression diagnosis and treatment in older Americans, conducting a trend analysis with data from the Medicare Current Beneficiary Survey (MCBS) from 1992 to 2005. The authors found that the proportion of older adults who received a depression diagnosis doubled, from $3.2 \%$ to $6.3 \%$ with rates increasing across all demographic subgroups.

7. Alegria M, Chatterji P, Wells K, Cao Z, Chen C, Takeuchi D, Jackson J, Meng X. Disparity in depression treatment among racial and ethnic minority populations in the United States. Psychiatr Serv. 2008;59(11):1264-72. doi:10.1176/appi.ps.59.11.1264.

8. Ruiz P. The persistence of disparities in mental health care. Editorial Psychiatric Services. 2008;59(11):1239.

9. Jeste DV, Alexopoulos GS, Bartels SJ, Cummings JL, Gallo JJ, Gottlieb GL, Halpain MC, Palmer BW, Patterson TL, Reynolds III $\mathrm{CF}$, Lebowitz BD. Consensus statement on the upcoming crisis in geriatric mental health. Arch Gen Psychiatry. 1999;56:848-53.

10. Hebert PL, Sisk JE, Howell EA. When does a difference become a disparity? conceptualizing racial and ethnic disparities in health. Heal Aff. 2008;27(2):374-82.

11. Commission on Social Determinants of Health, Closing the gap in a generation: Health equity through action on the social determinants of health. Final report of the commission on social determinants of health. Geneva: World Health Organization; 2008.

12. •• Smedley, B. D., Stith, A. Y., \& Nelson, A. R. (2003) Unequal Treatment: Confronting Racial and Ethnic Disparities in Health Care. Washington National Academies Press. Racial and ethnic disparities in health care are known to reflect access to care and other issues that arise from socioeconomic conditions. There is, however, increasing evidence that even after such differences are accounted for, race and ethnicity remain significant predictors of the quality of health care received. In this book a panel of experts explores how non-Whites experience the health care environment. Disparities in treatment are examined and aspects of the clinical encounter that may contribute to such disparities are investigated. The editors provide analysis of patients' and providers' attitudes, expectations, and behavior.

13. Joint Center for Political and Economic Studies (2010). Patient Protection and Affordable Care Act of 2010: Advancing Health Equity for Racially and Ethnically Diverse Populations. Washington, DC

14. Federal Interagency Forum on Aging-Related Statistics. (June, 2012). Older Americans 2012: Key Indicators of Well-Being. Washington, DC: U.S.

15. Emptage NP, Sturm R, Robinson RL. Depression and comorbid pain as predictors of disability, employment, insurance status, and health care costs. Psychiatr Serv. 2005;56(4):468-74. 
16. Greenberg PE, Stiglin LE, Finkelstein SN, Berndt ER. The economic burden of depression in 1990. J Clin Psychiatry. 1993;54(11):405-18.

17. U. S. Department of Health and Human Services. Mental health: Culture, face and ethnicity-a supplement to mental health. A Report of the Surgeon General. Rockville, MD: U.S. Department of Health and Human Services, Substance Abuse and Mental Health Services Administration, Center for Mental Health Services; 2001.

18. Sonnenberg CM, Deeg DJH, Comijs HC, van Tilburg W, Beekman ATF. Trends in antidepressant use in the older population: Results from the LASA-study over a period of 10 years. J Affect Disord. 2008;111:299-305.

19. Blazer DG, Hybels CF, Simonsick EM, Hanlon JT. Marked differences in antidepressant use by race in an elderly community sample: 1986-1996. Am J Psychiatry. 2000;57(7):1089-94.

20. Arean PA, Ayalon L, Hunkeler EM, Lin E, Tang L, Harpole L, Williams J, Hendrie H. Improving depression care for older minority patients in primary care. Medical Care. 2005;43(4):381-90.

21. Sorkin DH, Pham E, Ngo-Metzger Q. Racial and ethnic differences in the mental health needs and access to care of older adults in California. Journal of the American Geriatric Society. 2009;57:2311-7. doi:10.1111/j.1532-5415.2009.02573.x.

22. Trinh NT, LaRocca R, Regan S, Chang TE, Gilman SE, Fava M, Yeung A. Using the electronic medical record to examine racial and ethnic differences in depression diagnosis and treatment in a primary care population. Primary Health Care: Open Access. 2011;1:106. doi:10.4172/phcoa1000106.

23. Gonzalez HM, Tarraf W, Whitfield KE, Vega WA. The epidemiology of major depression and ethnicity in the United States. J Psychiatr Res. 2010;44:1043-51.

24. Case BG, Bertollo DN, Laska EM, Siegel CE, Wanderling JA, Olfson M. Racial differences in the availability and use of electroconvulsive therapy for recurrent major depression. J Affect Disord. 2012;136(3):359-65. doi:10.1016/j.jad.2011.11.026.

25. Givens JL, Houston TK, Van Voorhees BW, Ford DE, Cooper LA. Ethnicity and preferences for depression treatment. General Hospital Psychiatry. 2007;29:182-91.

26. Gonzalez HM, Vega WA, Williams DR, Tarraf W, West BT, Neighbors HW. Depression care in the United States: Too little for too few. Arch Gen Psychiatry. 2010;67(1):37-46.

27. Taylor SL, Lurie N. The role of culturally competent communication in reducing ethnic and racial healthcare disparities. American Journal of Managed Care. 2004;10:SP1-4.

28. Cooper LA, Roter DL, Johnson RL, Ford DE, Steinwachs DM, Powe NR. Patient-centered communication, ratings of care, and concordance of patient and physician race. Ann Intern Med. 2003;139(11):907-15.

29. Lewis-Fernandez R. Editorial: The cultural formulation. Transcultural Psychiatry. 2009;46:379-82.

30. Waite R, Killian P. Health beliefs about depression among African American women. Perspectives in Psychiatric Care. 2008;44 (3):185-95.

31. Connor KO, Lee B, Mayers V, Robinson D, Reynolds III CF, Albert S, Brown C. Attitudes and beliefs about mental health among older African American adults suffering from depression. Journal of Aging Studies. 2010;24:266-77.

32. •• Butler PD, Swift M, Kothari S, Nazeeri-Simmons I, Friel CM, Longaker MT, Britt LD. Integrating cultural competency and humility training into clinical clerkships: Surgery as a model. Journal of Surgical Education. 2011;68(3):222-30. doi:10.1016/ j.jsurg.2011.01.002. This article is a MEDLINE review of published literature regarding cultural competency and humility $(\mathrm{CCH})$ training in medical education. It is recognized that cultural competency is an essential strategy by which to address health care disparities. Through interviews conducted with faculty members from prominent medical schools the authors found that many academic medical institutions recognize the need for $\mathrm{CCH}$ training and have successfully integrated it into the first two years of their curricula.

33. Center for Workforce Studies (October, 2012). Recent studies and reports on physician shortages in the US. Retrieved on November 15, 2012. https://www.aamc.org/download/100598/data/ recentworkforcestudies.pdf

34. CDC Promotes Public Health Approach to Address Depression Among Older Adults (n.d.). Retrieved on October 14, 2012. http:// www.cdc.gov/aging/pdf/CIB_mental_health.pdf

35. Krause NM. Aging in the church: How social relationships affect health. West Conshohocken, PA: Templeton Foundation Press; 2008.

36. McFarland MJ. Religion and mental health among older adults: Do the effects of religion involvement vary by gender? Journal of Gerontology: Social Sciences. 2009;65B(5):621-30.

37. • Schnall E, Kalkstein S, Fitchett G, Salmoirago-Blotcher E, Ockene J, Tindle HA, Thomas A, Hunt JR, Wassertheil-Smoller S. Psychological and social characteristics associated with religiosity in Women's health initiative participants. J Relig Heal. 2012;51:20 31. The authors examined cross-sectional data from 92,539 postmenopausal participants in the Women's Health Initiative Observational Study, who responded to questions about religious service attendance, psychological characteristics and social support domains. The authors found that women attending services weekly during the last month were less likely to be depressed, more likely to be optimistic, and less likely to report social strain.

38. Johnstone B, Yoon DP, Cohen D, Schopp LH, McCormack G, Campbell J, Smith M. Relationships among spirituality, religious practices, personality factors, and health for 5 different faith traditions. J Relig Heal. 2012;51:1017-41.

39. Wittink MN, Joo JH, Lewis LM, Barg FK. Losing faith and using faith: Older African Americans discuss spirituality, religious activities, and depression. Journal of General Internal Medicine. 2009;24(3):402-7. doi:10.1007/s11606-008-0897-1.

40. Miranda J, Cooper LA. Disparities in care for depression among primary care patients. Journal of General International Medicine. 2004;19:120-6.

41. Shellman J, Granara C, Rosengarten G. Barriers to depression care for black older adults. J Gerontol Nurs. 2011;37(6):13-7.

42. Gullum, T. (2009). Intersection of Spirituality, Religion, and Intimate Partner Violence in the African-American Community. Retrieved November 16, 2012. http://www.idvaac.org/media/pubs/ TheIntersectionofSpirituality.pdf 